\title{
Navigating the adaptive cycle: an approach to managing the resilience of social systems
}

\author{
Brian D. Fath ${ }^{1,2}$, Carly A. Dean ${ }^{1}$ and Harald Katzmair ${ }^{3}$
}

\begin{abstract}
The concept of resilience continues to crescendo since the 1990s, touching on multiple fields with multiple interpretations and uses. Here, we start from its origins in systems ecology, framing the resilience concept explicitly in the adaptive cycle with the observation that resilient systems are ones that successfully navigate all stages of growth, development, collapse, and reorientation of this cycle. The model is explored in terms of the traps and pathologies that hinder this successful navigation, particularly when applied to socioeconomic organizations and decision-management situations. For example, for continuous function over the adaptive life cycle, a system needs activation energy or resources to grow, followed by adequate structure and complexity to maintain maturity. Implementation of crisis plans may avert collapse, but during catastrophe, the ability to improvise and re-orient will allow the system to emerge along a new cycle. We review the capacities, competencies, and cultures needed by these organizations, specifically, identifying that the needed resources are often cultivated in earlier stages, thus requiring consideration of the entire life cycle for success.
\end{abstract}

Key Words: adaptive cycle; collapse; development; growth; re-orientation; resilience; succession; thresholds

\section{INTRODUCTION}

\section{Resilience and the adaptive cycle}

Resilience thinking has gone through a number of iterations since its introduction as a technical ecological term by Holling in 1973 on spruce forest budworms. Important contributions were made building on these concepts within the ecological field, and expanding them to social-ecological systems (e.g., Gunderson 2000, Walker et al. 2002, 2004, Folke et al. 2005, 2010, Wilson et al. 2013), and then to social systems (e.g., Amundsen 2012, Radywyl and Biggs 2013, Ratter 2013). The term "resilience" has become popularized, with organizations building resilience concepts and strategies, from governments managing for healthcare issues (Thomas et al. 2013), to general resilience of cities (Geneske 2013), and resilience approaches in sociology (Edson 2012). The concept of resilience continues to be applied at larger scales in a greater variety of fields. This universal applicability suggests that resilience is an inherent property of systems, although a clear approach to managing resilience is still lacking (Redman 2014, Standish et al. 2014).

The Holling (1986) lazy-eight adaptive cycle model (Fig. 1) is a powerful and useful metaphor of system dynamics that includes four stages: growth $(\mathrm{r})$, equilibrium $(\mathrm{K})$, collapse $(\Omega)$, and reorientation $(\alpha)$. This extends the traditional successional logistic curve $(\mathrm{r} \rightarrow \mathrm{K})$ to include explicitly the collapse and reorganization phases. However, we use a modified version (Fig. 2), which more accurately represents a system's dynamics. This version, from Burkhard et al. (2011), has two main deviations from the original Holling figure. First, it is rotated $45^{\circ}$ to correct for the incongruous pattern in the original $\Omega$ quadrat in which the abscissa values inexplicably increased at the end of the collapse stage prior to emergence from the reorganization phase. In the revised version, the system experiences a monotonic collapse from its K-phase height through to the beginning of the reorganization stage. Second, during the successional growth and development stage $(\mathrm{r} \rightarrow \mathrm{K})$, the upward trajectory is objectively not monotonic on a small scale (represented by the squiggly path), although an overall upward trajectory is traced. We use this amended figure as the starting point for an expanded definition of resilience. In our terms, resilience is the capacity to successfully navigate ALL stages of the complex adaptive cycle $(r, K, \Omega$, and $\alpha$ ). This approach builds on the earlier seminal work of Walker et al. (2002), who considered the role of stakeholder participation in resilience management using the adaptive cycle metaphor. Using this holistic perspective, we identify dominant system properties needed at each stage, as well as systemic pathologies resulting in traps in the adaptive cycle, and then explore principles and variables important to systems navigating the adaptive cycle and cultivating more resilient social systems.

Fig. 1. Adaptive cycle. The tail labeled " $x$ " indicates the potential for the system to undergo a regime shift. From Panarchy by Lance Gunderson and C.S. Holling. Copyright (C) 2002 Island Press. Reproduced by permission of Island Press, Washington, DC.

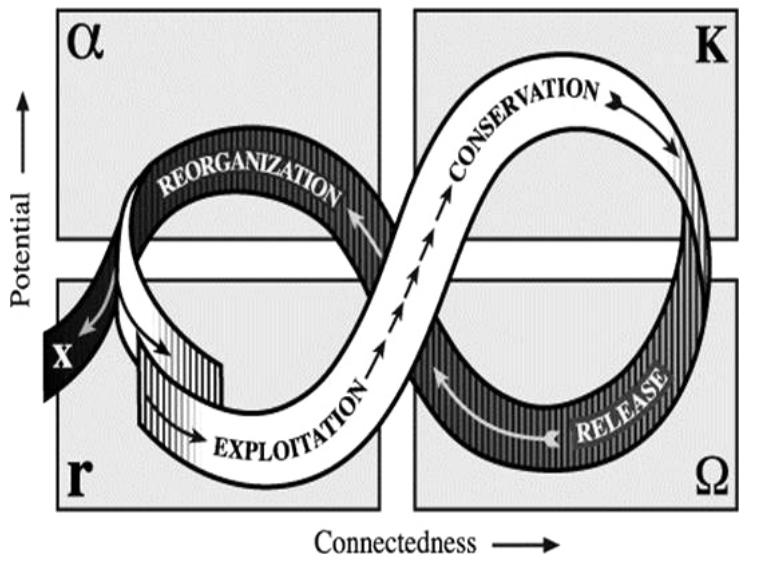

${ }^{1}$ Advanced Systems Analysis, International Institute for Applied Systems Analysis, ${ }^{2}$ Department of Biological Sciences, Towson University, ${ }^{3}$ FAS. research 
Fig. 2. Adaptive cycle as modified by Burkhard et al. (2011).

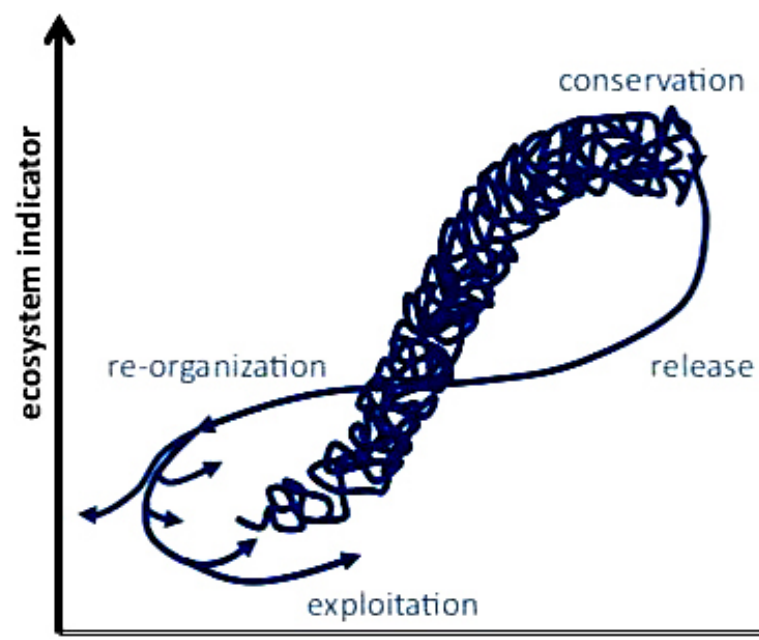

number of connections

Resilience is often associated with a system's ability to recover from a disturbance. This is in contrast to resistance, which is a system's ability to withstand a disturbance with little deformation. Here, we combine the resilience concept with the entire adaptive cycle to provide a comprehensive definition that applies to all stages of a system's dynamics. This approach aligns complementarily (but not identically) with the concept of sustainability (Fath 2015), defined as a system's ability to persist over time. In other words, sustainability occurs over an infinite time horizon in which the objective is to persist and maintain system function. An apt analogy for sustainability is taken from Carse (1987), who referred to finite and infinite games: the former have the goal of winning, the latter a goal of continuing to play the game. Here, resilience is a necessary but not sufficient feature that promotes system sustainability, i.e., continuing to play the game. We next expand on this definition and identify key principles of the resilience of social systems.

\section{CONCEPTS}

Principles of resilience in social systems

The core concepts of this new, combined model applied to social organizations are given in Fig. 3. These concepts include the four stages of the adaptive cycle: $\mathrm{r}$ : new beginning and growth; $\mathrm{K}$ : conservation and status quo ante; $\Omega$ : dissolution and confusion; and $\alpha$ : reorganization and innovation. It is notable that there are many small-scale adaptive cycles embedded in the growth and development ( $\mathrm{r}, \mathrm{K}$ ) stages, representing modular experimentation within the overall upward system trajectory. An important distinction is made during the equilibrium phase between two types of perturbations: those crises that are contained within system's vertical range of tolerance, considered as $\mathrm{K}_{\text {lim }}$; and those that exceed the threshold and propel the system toward dissolution. The objective here is not to dwell on identification of the threshold but rather to consider the possibilities for both scenarios.
Given that a resilient system is one that can navigate all stages of the adaptive cycle ( $\mathrm{r}, \mathrm{K}, \Omega$, and $\alpha$ ), we look more closely at the key features for success through each stage. Table 1 lists some of the needed capacities for success in each stage.

Table 1. Key features for the success of a system (entry points).

\begin{tabular}{ll}
\hline \hline Needed capacity & Requirement for success \\
\hline Capacity to grow $(\mathrm{r})$ & $\begin{array}{l}\text { Activation energy } \\
\text { Capacity to develop }(\mathrm{K})\end{array}$ \\
$\begin{array}{l}\text { Selforganized to store information and } \\
\text { capital }\end{array}$ \\
Capacity to survive $(\Omega)$ & $\begin{array}{l}\text { Improvise to maintain vital functions } \\
\text { Learn and forgive to reorient }\end{array}$ \\
\hline
\end{tabular}

$r$-stage

The goal of the r-stage is growth; the trap of this stage is called the "poverty trap" (Gunderson and Holling 2002). A system in the r-stage has successfully reoriented post-crisis and now seeks the activation energy for rapid growth and development. In the study of the adaptive cycle, sufficient activation energy refers to the availability of resources and information to enter the growth stage. The system invests those resources during the growth stage to build structure with diverse nodes and flows. The poverty trap occurs when a system cannot access enough activation energy to reach a state where positive feedbacks drive growth internally. Additionally, with the goal of growth, the system at this stage must develop a configuration that is simple enough to scale, a characteristic that must be balanced with the cultivation of a level of internal complexity that will result in autocatalysis and selfsustainability (Ulanowicz et al. 2009, Fath 2015). The r-stage is often marked by abundant resources and entrepreneurial leadership. The system is brimming with untapped and uncommitted potentiality (Bateson 1972). Reconfiguration from unformed supplies to coupled agencies and capital is essential to system maturation. Here, innovation comes from plenty, rather than from constraint, as is the case in the K-stage. Once kickstarted along a growth trajectory, many resource flows are available for experimentation. In the r-stage, network connections are established, and trust and dependencies are built. Further, in this stage, the dynamics between feedbacks are essential for the system to enter the K-stage.

K-stage

The K-stage, or equilibrium-stage, is about controlled development. Entering the K-stage is about making a transition from quantitative increase to qualitative indicators. In ecological systems, this is equivalent to climax ecosystem states in which the resource inflows in terms of gross primary production are consumed by process maintenance in terms of respiration (production $=$ respiration) such that excess resources are unavailable for growth. A mature system in the K-stage dynamically performs at a high level of activity while the macroscale indicators display stable functions. The resource constraints may spur innovation through co-creation of networks and information flows that are able to use the input flows more effectively to maintain high levels of structure and organization (Fath et al. 2004). This organization manifests itself as the internal storage of information and capital (acquired in the growth phase). At this stage, negative feedback cycles dominate over positive 
Fig. 3. Adaptive cycle applied to social systems. Stages in this cycle are similar to ecological stages, from new growth to status quo, to confusion, and innovation. The differentiation between crises that remain within the threshold and those that lead to dissolution are indicated by the vertical range of tolerance.

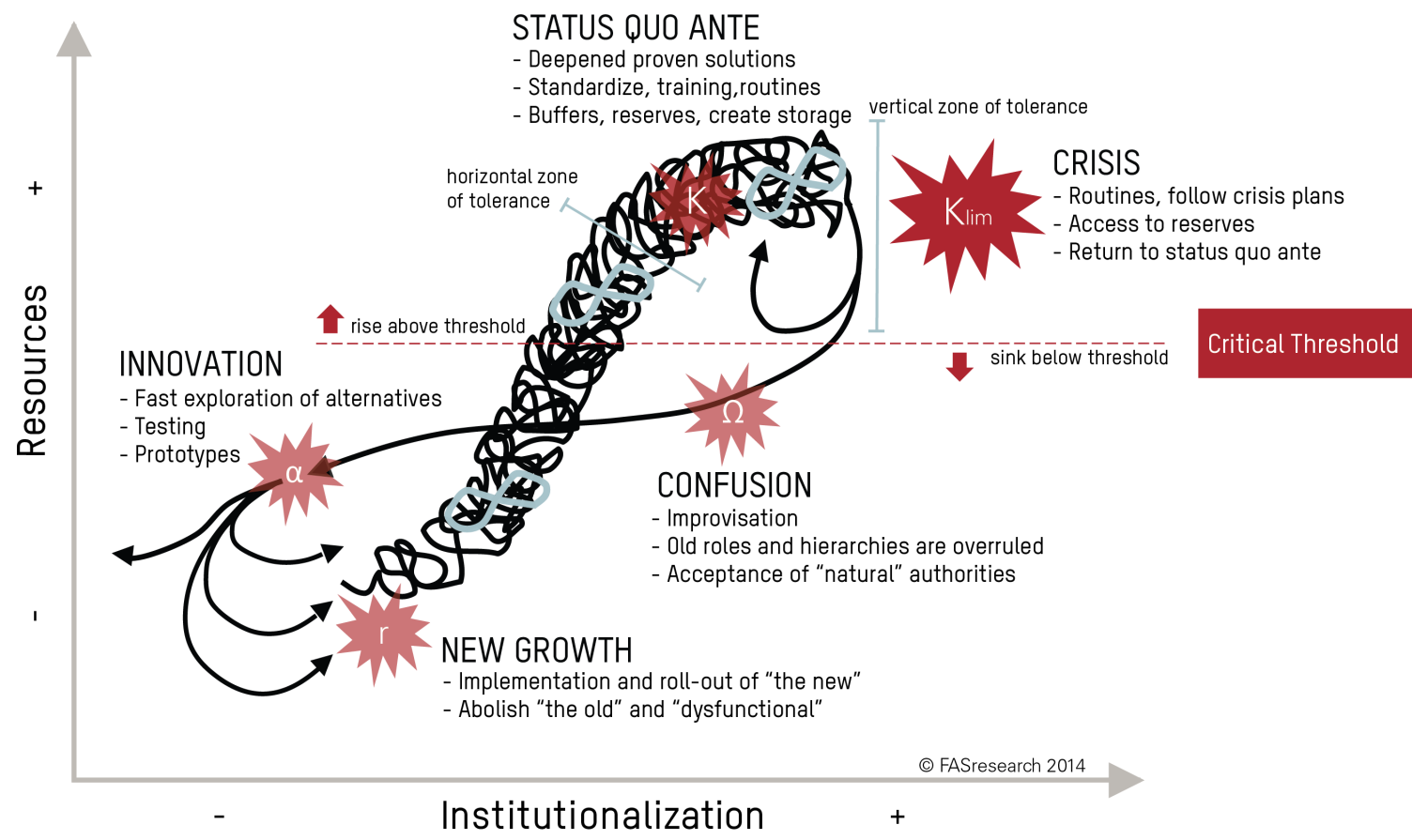

feedbacks. When positive feedbacks exceed negative feedbacks to the extent of exhausting resources, the system trajectory can overshoot thresholds, sabotaging future system function.

In addition to the possibility of overshoot, the trap here is the "rigidity trap" (Gunderson and Holling 2002). The rigidity trap occurs when a system becomes so refined in its processes that there is little room for further innovation. Characteristics of a rigid system include very few key nodes with a high concentration of influence, and low diversity both in nodes and pathways, i.e., the system has reduced its entropy so effectively that there is no longer any "ontic openness" (Nielsen and Ulanowicz 2011) or white space for novelty to emerge. Additionally, a rigid system is brittle and vulnerable to disturbance because of reduced diversity and inability to self-organize. While we posit that the navigation of the full adaptive cycle indicates a resilient system (and the $\Omega$-stage is inevitable), it is also often desirable to extend the $\mathrm{K}$-stage of the adaptive cycle, not in the form of a trap, but rather with continued development, as expressed as an indicator of system performance. In this manner, the system may persist through a crisis if $\mathrm{K}_{\mathrm{lim}}$ is not exceeded, which can be endogenously influenced by the preparedness and response of the actors.

$\Omega$-stage

The test of a system in the $\Omega$-stage is its capacity to survive in the face of extreme disturbance or disordered collapse. A system must maintain vital functions throughout the crises. In human organizations, it is often up to leadership, both assigned and assumed, to identify and prioritize what that means. One of the ways that the diversity maintained through small-scale disturbances contributes to the resilience of the system is by cultivating a large stock of resources from which it can pull during a crisis, both in terms of organizations and their relationships, which is essential for leadership to emerge during the $\Omega$-stage. Emergent leadership occurs when actors not tasked with leadership roles informally assume key positions during crisis. In a study of New York City's disaster response to 9/11, even secondary and tertiary coordinators played important roles in maintaining vital functions (Schweinberger et al. 2014). Crisis coordinators that emerge during the $\Omega$-stage are an example of "unpredictable combinations of [de novo entities] with existing components that can suddenly establish new domains of influence, opening an entirely new set of adaptive pathways" (Gunderson and Holling 2002:403). Thus, emergent coordinators set the stage for learning, adaptive capacity, and reorientation of a resilient system successfully navigating through the cycle in the future. Failure to survive this stage results in a complete break of the system cycle. The alternative is the "dissolution trap".

$\alpha$-stage

Recognizing that a complex adaptive system can never return to the precise structure, function, and feedback as before (Folke 2006), Bellwood et al. (2004) wrote that resilience authors prefer the terms renewal, regeneration, and reorganization instead of the term recovery. We add to this concept the trajectory-based idea of reorientation such that the system escapes from the freefall of the collapse phase along a new trajectory for future development. Success depends on the ability to tap into the system characteristics outlined below to continue navigating the adaptive cycle. The $\alpha$-stage has the goal function of reorientation. 
The idea of system identity comes into play at the $\alpha \rightarrow r$ transition under the assumption that typical resilience thinking directs us to identify whether the period following the $\alpha$-stage represents a new or the same regime, that is to say, whether it passed the critical threshold and operates under a new set of rules with different structure, function, and feedbacks. However, this threshold can be difficult to define based on that interpretation of resilience, especially because similar looking systems may not be the same because of having experienced the disturbance (Folke 2006). Therefore, as discussed above, we propose the paradigm shift that resilience is less characterized by systemic properties and rather operates on the context of the system. Within social organizations, to be successful, managers must clarify the goal or direction or focus of the system (Scheffer et al. 2002, Pelling et al. 2008, Henry and Ramierez-Marquéz 2012) because resilience lies in the ability to move out of the $\alpha$-stage and into a new r-stage trajectory while still providing a certain level of net social utility (Carpenter et al. 2001). This view of resilience tends to avoid some of the normative beliefs associated with maintaining particular aspects of social systems and aligns with the idea of "perverse resilience" (Phelan et al. 2013) in that the resilience of components or nested subsystems can have resilience that counters the resilience of the larger system. That is to say, the persistence of particular components is irrelevant, so long as the system continues to navigate the adaptive cycle.

How does a system continue to produce the same kind of net social utility even after key nodes or links have been damaged or lost through the disturbance? The answer lies in systemic memory, which is largely cultivated in the previous stages of the cycle. In biological systems, memory is in seed banks stored in soil and neighboring communities that can help repopulate a disturbed area; these are both forms of capital that were stored during the front loop of the adaptive cycle. To reorient after crises, a system must reorganize and access capital, that is, stored emergency capital and pathways for access established prior to the crises. These pathways, or node relationships, are maintained through disturbance by the property of transitivity (Schwienberger et al. 2014) wherein even though an edge is removed that links two nodes, there are alternative pathways that allow each organization to reach every other node directly or indirectly through a third node. Memory in a system is retained if a system has high modularity (Biggs et al. 2012), which helps to prevent failure from penetrating all aspects of system function (May et al. 2008, Levin et al. 2013). The success of navigating through the fast-moving $\alpha$-stage is largely a function of system development and decisions made in prior stages. Being unable to reorient leaves the system in the "vagabond trap". Having too many nodes and links lost to access system memory, i.e., circling compassless, without moving into the r-stage of growth, is largely preventable through prudent systemic development during the $\mathrm{r}$ and $\mathrm{K}$ stages with the awarenss of the inevitability of disturbance.

\section{RESULTS}

\section{Pathologies that inhibit successful navigation along the complex adaptive cycle}

While each stage of the resilience cycle has a particular capacity and trap to overcome, it is informative to explore the various barriers that may arise. We refer to these impediments as pathologies or ailments that may interfere with successfully navigating the adaptive cycle. Furthermore, we consider the dual nature that for continuous "play" of the cycle there must be key features at both the entry and exit points. These pathologies (Table 2) are described here briefly and are to be considered while referencing the adaptive cycle.

Table 2. Key entry and exit points for each stage of the adaptive cycle.

\begin{tabular}{lll}
\hline \hline Stage & Entry & Exit \\
\hline $\mathrm{r}$ & $\begin{array}{l}\text { Poverty trap: no } \\
\text { activation energy, no } \\
\text { scaling }\end{array}$ & $\begin{array}{l}\text { Overshooting ("forever young"), } \\
\text { relentless resource acquisition }\end{array}$ \\
$\mathrm{K}$ & $\begin{array}{l}\text { Lack of internal } \\
\text { complexity (right } \\
\text { buffers, redundancies, } \\
\text { connections) }\end{array}$ & $\begin{array}{l}\text { Perpetuation of status quo } \\
\text { through cannibalism; rigidity trap } \\
\text { (loss of connection to the outside) }\end{array}$ \\
$\Omega$ & $\begin{array}{l}\text { Subsidize rigid systems } \\
\text { Self-victimization } \\
\text { mentality }\end{array}$ & $\begin{array}{l}\text { Inability to improvise } \\
\text { Lack of direction (no scale, no } \\
\text { new orientor) }\end{array}$ \\
\hline
\end{tabular}

Box 1: Key preparedness features that must be cultivated in each stage of the adaptive cycle. For example, to escape the vagabond trap requires self-organization, access to stored capital, memory, and modularity, each developed in the following stages, respectively: $r, \mathrm{~K}, \Omega$, and $\alpha$.

Escaping the vagabond trap: $\alpha$-stage

1. Self-organization (r): The extent to which the system can restructure social networks and develop new organizations from within.

2. Access to stored capital $(\mathrm{K})$ : The access to emergency resources in the form of natural, built, human, economic, and social capital during and post-crisis.

3. Memory $(\Omega)$ : Remembering both past crisis experiences and past successes.

4. Modularity $(\alpha)$ : Sets of densely connected nodes loosely connected to other subsets of nodes.

Escaping the poverty trap: r-stage

1. Positive feedbacks (r): A change in a particular variable, process, or signal reinforces subsequent changes of the same type.

2. Bilateral information flows (K): Information flowing in both directions of system hierarchy.

3. Emergent leadership $(\Omega)$ : Emergence of and collaboration with organizations not originally tasked with a particular crisis response.

4. Adaptive capacity ( $\alpha$ ): Recognizing learning experiences and using the opportunity to make adjustments to behavior.

Escaping the rigidity trap: K-stage

1. Negative feedbacks (r): Structural characteristics that regulate the rate of growth.

2. Maintain diversity $(\mathrm{K})$ : Diversity in function and response of components and their relationships. 
3. Small-scale disturbances $(\Omega)$ : The frequency and intensity of noncrisis disturbances.

4. Buffer capacity $(\alpha)$ : Stored capital and redundancies within the system.

Escaping the dissolution trap: $\Omega$-stage

1. Reduce fault cascade (r): The ability to prevent crises from spreading throughout the system through early detection and organizational structure.

2. Cohesive leadership (K): Key actors that back growth financially and spread information rapidly.

3. Maintain vital functions $(\Omega)$ : Identifying and maintaining functions that are essential to the continuation of a minimum level of social utility.

4. Improvisation $(\alpha)$ : Suspending prescribed roles in response to immediate needs.

As stated above, entry into the r-stage is blocked under the poverty trap when sufficient energy and resources are unavailable to kickstart the positive feedbacks needed to initiate growth. Also, it is necessary that actors have the knowledge to perform the needed action from past memory or new training. Incapability to exit from the r-stage occurs if the system's negative feedbacks cannot control the growth and the system overshoots its carrying capacity. Relentless resource acquisition then is necessary to maintain both the high level of complexity as well as continued growth. Systems dominated by the growth paradigm suffer from an inability to transition smoothly from r-stage to K-stage. Furthermore, systems are unable to enter to the $\mathrm{K}$-stage if they lack the internal complexity required to maintain structures in the equilibrium phase. They resist exiting the K-stage through "pulling out the stops" to maintain the status quo, even if it means cannibalizing the structures themselves to acquire the accustomed resource flows in the growth stage. This is akin to feeding the masses by eating the seed corn, rather than sustainably planting it in anticipation of next year's harvest. There are two reinforcing factors at work here. First, it is a natural reaction for the actors in the system to attempt to delay the collapse by whatever means possible. In this manner, the disturbance does not push the system beyond the $\mathrm{K}_{\lim }$ threshold such that it regroups to essentially its previous manifestation. Second, these actions paradoxically further act to undermine the sustainability of the system, only providing transitory reprieve from the inevitable emergence of the $\Omega$-stage. Furthermore, these actions may weaken a system's ability to respond after collapse. Scale issues also obscure the understanding of the equilibrium phase because multiple small-scale disturbances may effectively delay a big collapse. As stated above, innovation under a fast-time, small-space scale may surface as a way to elude the rigidity trap.

A management approach that chooses to prop up and subsidize rigid structures prohibits entry in the $\Omega$-stage, exhibiting yet another potential pathology. The idea of a controlled descent (e.g., Odum and Odum 2001) has not seriously entered the dialog. Exit from the $\Omega$-stage can be blocked if the system is unable to improvise and survive during the disruptions. One may say that this stage truly represents an existential threat to the system, so all attention must be focused on survival. Preparedness and emergent leadership can help ensure successful passage through this stage. Overlooking the case of complete disappearance of the system, entry into the $\alpha$-stage is predicated on the ability to reassemble the pieces. To do so in a social or business setting, there must be a psychological acceptance of the new situation that permits those affected to forgive and let go (not the same as forgetting) of the circumstances that brought about the collapse to avoid wallowing with a paralyzing, self-victimization mentality. Once the regrouping has begun, it is still necessary to find new orientation to exit the $\alpha$ stage successfully. The pathologies described here provide a framework for anticipating the traps to avoid as well as considerations for preparedness. Another key insight from this is that preparedness must be cultivated during each of the stages in anticipation of the inevitable transition to subsequent stages. Below, we consider the aspects most relevant to becoming prepared.

\section{Cultivating preparedness: competences, resources, and cultures} We reviewed resilience literature to identify system principles that have been indicated as important to resilience, filtered by which principles can apply specifically to social organizations. We propose where in the adaptive cycle these principles are most important in terms of successful pathway navigation, avoiding traps, and preparing for enhanced systemic performance. In the literature, we pulled out many different variables that are discussed when assessing the resilience of anything from the resilience of spruce forests to budworms (Holling 1973), political systems to climate change (Phelan et al. 2013), and rural communities to demographic decline (Amundsen 2012). The list of terms associated with the resilience literature from diverse disciplines is multitudinous, from which we distill key terms aligned with a particular phase of the cycle and indicate when operative cultivation is most likely to occur. Based on the definition that a resilient system is one that successfully navigates through each stage of the adaptive cycle, it is thus the ability to escape traps at each stage that results in a resilient system. We identify the resilience principles that are most essential to escaping that stage's trap and note that the capacity to manage the trap is cultivated in all stages, not just the crisis stage or immediately preceding stage (Box 1).

In the domain of social systems, in particular business management, it is useful to consider specifically these features in terms of the competences, resources, and culture that promote the successful navigation through the adaptive cycle. Examples of these concepts are given in Table 3. Summary from practical business applications are identified in Table 4.

\section{SPECULATION}

The original applications of the adaptive cycle and resilience concepts stemmed from Holling's work in systems ecology. The ideas have been co-opted and modified for a variety of uses over the years, namely because they provide a deeper understanding of a broad range of systems, including social and business organizations. While ecosystems are complex, human agency adds a less understood and therefore less predictable component, but also adds potential responsive dynamic preparedness and management that is otherwise not found in ecological systems. 
Table 3. Competences, resources, and culture aiding navigation through the adaptive cycle applied to business management.

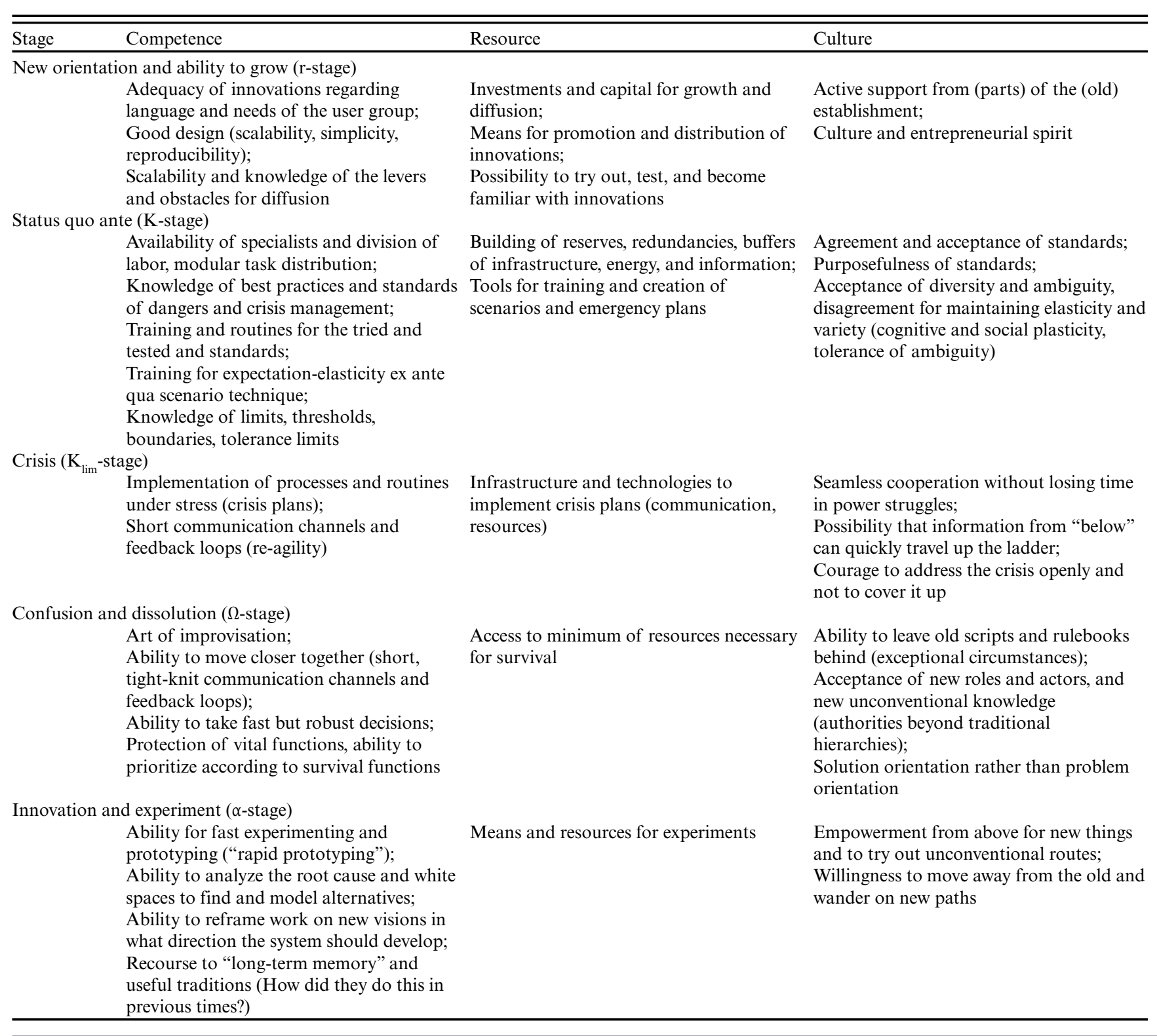

Further, the interaction between temporal scales adds another dimension because different individuals who make up the system have different views on what the present and future mean and how to value them (i.e., discount rate, ability to anticipate consequences; Holdschlag and Ratter 2013); differentiating spatial scale also becomes difficult because individuals operate on and have different interpretations of diverse scales (what is local to one person/group/organization might be different to another that acts within the same system). Carpenter et al. (1999) identify "key characteristics of nonlinearity and complexity in socioeconomic systems: many individual, boundedly rational agents or institutions making decisions (using formal or informal rules) and learning about a world they co-create."

Therefore, there are important points that denote successful navigation throughout each stage of the adaptive cycle, as well as pathologies that indicate either traps within that cycle or a trajectory toward undesirable performance levels. Ultimately, resilience is linked to some dynamic between slow variables that represent the underlying structure of the system and fast variables that reflect dynamics in the present (Carpenter et al. 2001, Gunderson and Holling 2002, Biggs et al. 2012); some resonance between these scaled variables (i.e., panarchy) moves the system through the cycle and ultimately leads to a tipping point. Each ecological system is different in terms of fast and slow variables, but we think that it is social cohesion in social systems that represents the interplay between fast and slow variables. We propose that social cohesion can help in understanding the relationship between fast and slow variables and can provide some insight into the resilience of social networks. Just like phosphorous in the lake system, social cohesion represents the 
Table 4. Examples from business organizations of successfully navigating each phase.

\begin{tabular}{|c|c|c|c|c|}
\hline Stage & Competence & Resources (capacities) & Culture & Other \\
\hline \multicolumn{5}{|c|}{ Escaping the vagabond trap: $\alpha$-stage } \\
\hline & $\begin{array}{l}\text { Fast iterations of prototypes and } \\
\text { short feedback loops instead of } \\
\text { pursuing perfection; } \\
\text { Co-creation and co-development } \\
\text { with customers to meet later } \\
\text { needs ("open innovation") }\end{array}$ & $\begin{array}{l}\text { Curious, passionate, open- } \\
\text { minded people with room for } \\
\text { experiment and play }\end{array}$ & $\begin{array}{l}\text { Design thinking, culture of } \\
\text { prototyping, heterogeneous, } \\
\text { transdisciplinary teams } \\
\text { (Skunkworks, linked diversity } \\
\text { groups); } \\
\text { Culture of "directional } \\
\text { correctness" rather than "truth" } \\
\text { and "optimum") }\end{array}$ & $\begin{array}{l}\text { Generally having a (rough) scale } \\
\text { and sorting criteria to rank and } \\
\text { weed out variations among ideas } \\
\text { and prototypes }\end{array}$ \\
\hline \multicolumn{5}{|c|}{ Escaping the poverty trap: r-stage } \\
\hline & $\begin{array}{l}\text { Do the right things right } \\
\text { (strategy skills); } \\
\text { Know and meet needs of } \\
\text { customers; } \\
\text { Know how to scale (simplicity of } \\
\text { solution) }\end{array}$ & $\begin{array}{l}\text { Attract the best people; } \\
\text { Capital to invest in growth ("You } \\
\text { cannot starve yourself into } \\
\text { growth"); } \\
\text { Having the right partners and } \\
\text { networks }\end{array}$ & $\begin{array}{l}\text { Flexible, generalist mindset; } \\
\text { Support from parts of the } \\
\text { established elites in the market }\end{array}$ & $\begin{array}{l}\text { Generally having a product/ } \\
\text { service that taps into a gradient } \\
\text { that others perceive as valuable }\end{array}$ \\
\hline \multicolumn{5}{|c|}{ Escaping the rigidity trap: $\mathrm{K}$-stage } \\
\hline & $\begin{array}{l}\text { Have an understanding of the } \\
\text { danger of too much success } \\
\text { ("lock in"); } \\
\text { Lack of "slack" due to hyper- } \\
\text { efficiency and too much dealing } \\
\text { with bureaucratic rules (self- } \\
\text { absorption) }\end{array}$ & $\begin{array}{l}\text { Access to financial markets to } \\
\text { fund mergers and acquisitions as } \\
\text { a means to renew frozen, rigid } \\
\text { organizations; } \\
\text { Maintain flexible overhead to } \\
\text { fund experiments and noncore } \\
\text { business exploration }\end{array}$ & $\begin{array}{l}\text { Maintain a culture of openness, } \\
\text { playfulness and curiosity; } \\
\text { Rotational teams; } \\
\text { Cross-scale collaboration; } \\
\text { Strong culture of continuous } \\
\text { learning (flow of sense, } \\
\text { understand, and respond) }\end{array}$ & $\begin{array}{l}\text { Have an understanding that } \\
\text { intentional destabilization is } \\
\text { mandatory to keep the } \\
\text { organization agile and flexible } \\
\text { (equilibrium = death to an } \\
\text { organization) }\end{array}$ \\
\hline \multicolumn{5}{|c|}{ Escaping the dissolution trap: $\Omega$-stage } \\
\hline & $\begin{array}{l}\text { Capability to improvise and be } \\
\text { creative on-the-spot; } \\
\text { Ability to tell a strong story } \\
\text { about future, alternative routes } \\
\text { of development }\end{array}$ & $\begin{array}{l}\text { Having minimum access to } \\
\text { credit; } \\
\text { Understand who core assets and } \\
\text { core employees are and be able } \\
\text { to keep them }\end{array}$ & $\begin{array}{l}\text { Strong storytelling ("hold on } \\
\text { stories"), charismatic leadership; } \\
\text { Strong bonds among core team; } \\
\text { "Letting go" as part of } \\
\text { organizational culture (rituals) }\end{array}$ & $\begin{array}{l}\text { Have an understanding in times } \\
\text { of hardship that de-structuring is } \\
\text { not a pathology but a necessity } \\
\text { for renewal and future growth } \\
\text { and development }\end{array}$ \\
\hline
\end{tabular}

underlying relationships across space and time between different components or nodes and processes or links that result in the current state of the system. Social cohesion can represent a shared vision and identity, as well as priority of stakeholders that are at the same time based on deeply rooted cultural beliefs (slow variables) and on quickly shifting perspectives and priorities (fast variables) of a diverse yet highly integrated society. The explicit role for social cohesion is a question for future research. This understanding can give some insight into how a system functions and on how to increase the resilience of a system.

Bilateral information flows, that is, bottom-up as well as the traditional top-down forms of information flow, are an indicator of resilient systemic development and successful navigation through the adaptive cycle. Several researchers have commented on the role of multilevel interactions in enhancing resilience (Gunderson and Holling 2002, O'Brien and O'Keefe 2010, Robinson and Berkes 2011). These interactions and learning processes can result in leadership that recognizes the structure of a complex adaptive system and thus understands how the system functions at different levels. When leadership remains connected in this way, renewal occurs internally while overall structure is maintained. Bilateral information flows contribute to the adaptive capacity of an organization and reflect the human agency of social systems. When a leader grasps the structure and feedbacks of the system's different nested scales, that is, information successfully flows from the bottom up, they can recognize where within the adaptive cycle different sub-systems lie and how their trajectories affect the other sub-systems and the system as a whole. It is then the responsibility of the leadership to ensure that systems at lower scales have the resources and guidance to continue their trajectory in a way that is beneficial to the rest of the system (support positive feedbacks). For instance, as was found in a study of political systems, when certain subsystems such as unaffiliated or disengaged actors and groupings, counter-hegemonic sustainability forces, and the fossil fuel historical bloc have perverse resilience (that which conflicts with the resilience of the larger system; Phelan et al. 2013), a successful leader can initiate control measures to redirect trajectories or avoid overshoots (support negative feedbacks).

One potential variable to examine is the effect of small-scale disturbances in the K-stage. Small-scale disturbances (or what some have referred to as "shock therapy"; Gunderson and Holling 2002) should be encouraged (Biggs et al. 2012) because they contribute to the adaptive capacity of the system and its ability to innovate. These small-scale disturbances can promote the use of alternative pathways to transfer information throughout the system, i.e., the transitivity of the system (Schweinberger et al. 2014) by seeking opportunities in the way sub-systems interact on different time scales, i.e., the asynchrony (Isbell et al. 2009, Hector et al. 2010) of different systems. Small-scale disturbances represent multi-level interactions that promote learning of the system and allow upward flows of information through the 
panarchy, that is, through smaller, faster sub-systems to the larger, slower system, to maintain and cultivate development even at the $\mathrm{K}$-stage. In the classic example of frequent fires in a grassland ecosystem, these small-scale disturbances help to promote diversity by limiting any one node or link from becoming too strong, thus preventing the system from becoming too brittle. Small-scale disturbances encourage the participation of diverse actors that can contribute to the system in a variety of ways, beyond those of the key actors and leaders. Encouragement and creation of these small-scale disturbances can allow internal feedback resulting in increased adaptive capacity when the system is confronted with large-scale disturbances (Gunderson and Holling 2002). After all, a trapped system occurs when the upward flow of information is curtailed (Gunderson and Holling 2002). By understanding small-scale disturbances within a system, we can gain some information about their frequency and effect within social organizations as a larger part of the system as well as within the system itself and to what extent they see these as learning experiences that contribute to the preparedness and resilience of the larger system.

\section{CONCLUSIONS}

Based on the concepts and guidelines presented here, we argue for the integration of a continuous model of resilience throughout stages of growth, development, collapse, and re-organization. Because complex adaptive systems are always learning and thus are never able to return to the same pre-disturbance state, we propose that a resilient system is not one that necessarily must fall back to the same cycle represented by Holling's lazy-eight as before the disturbance (the $\alpha \rightarrow r$ transition), but rather is one that is able to navigate successfully through each stage of the cycle, adopts a new regime that shares important features of the previous regime, and continues to satisfy a set of goals as defined by members within that organization. In congruence with the more myopic focus on engineering resilience and visualization of the system as a logistic curve (or front loop with only the $\mathrm{r} \rightarrow \mathrm{K}$ stages), managers likely focus on the traps, critical thresholds, and principles associated with the front loop of the adaptive cycle. These traps are summarized as the poverty trap (r), rigidity trap $(\mathrm{K})$, dissolution trap $(\Omega)$, and vagabond trap $(\alpha)$. Entry and exit of the different stages can also be facilitated by awareness and avoidance of stage-specific pathologies (Table 2). We propose that application of this conceptual model can aid in managing the resilience of a social or business organization to understand the links between those principles and the ability to avoid traps and continue the navigation throughout all four stages of the adaptive cycle. It is also important to note that although we suggest that these principles are most highly developed in a particular stage and are then most influential to escape a particular trap, they will influence the behavior of the system throughout all stages to some extent, and can be drawn upon by the system outside of the framework indicated. We thus acknowledge that the boundaries between one stage and another may be fuzzy, and that at different times, systemic properties may be accessed in a dynamic environment.

Responses to this article can be read online at: http://www.ecologyandsociety.org/issues/responses. php/7467

\section{Acknowledgments:}

We appreciate helpful comments on a previous version of the manuscript from Sally Goerner, Tiina Häyhä, and Ali Kharazi. Funding support for this project was provided by Austrian Security Research Programme (KIRAS). The project Re.M-Austria (or Resilience Monitor Austria) is funded by the Austrian security research programme KIRAS of the Federal Ministry for Transport, Innovation and Technology (bmvit).

\section{LITERATURE CITED}

Amundsen, H. 2012. Illusions of resilience? An analysis of community responses to change in northern Norway. Ecology and Society 17(4): 46. http://dx.doi.org/10.5751/ES-05142-170446

Bateson, G. 1972. Steps to an ecology of mind: collected essays in anthropology, psychiatry, evolution, and epistemology. University of Chicago Press, Chicago, Illinois, USA. http://dx.doi. org/10.7208/chicago/9780226924601.001.0001

Bellwood, D. R., T. P. Hughes, C. Folke, and M. Nyström. 2004. Confronting the coral reef crisis. Nature 429:827-833. http://dx. doi.org/10.1038/nature02691

Biggs, R., M. Schlüter, D. Biggs, E. L. Bohensky, S. BurnSilver, G. Cundill, V. Dakos, T. M. Daw, L. S. Evans, K. Kotschy, A. M. Leitch, C. Meek, A. Quinlan, C. Raudsepp-Hearne, M. D. Robards, M. L. Schoon, L. Schultz, and P. C. West. 2012. Toward principles for enhancing the resilience of ecosystem services. Annual Review of Environment and Resources 37:421-448. http:// dx.doi.org/10.1146/annurev-environ-051211-123836

Burkhard, B., B. D. Fath, and F. Müller. 2011. Adapting the adaptive cycle: hypotheses on the development of ecosystem properties and services. Ecological Modelling 222(16):2878-2890. http://dx.doi.org/10.1016/j.ecolmodel.2011.05.016

Carpenter, S., W. Brock, and P. Hanson. 1999. Ecological and social dynamics in simple models of ecosystem management. Conservation Ecology 3(2): 4. [online] URL: http://www.consecol. org/vol3/iss2/art4/.

Carpenter, S., B. Walker, J. M. Anderies, and N. Abel. 2001. From metaphor to measurement: Resilience of what to what? Ecosystems 4(8):765-781. http://dx.doi.org/10.1007/s10021-001-0045-9

Carse, J. P. 1987. Finite and infinite games: a vision of life as play and possibility. Ballantine Books, New York, New York, USA.

Edson, M. C. 2012. A complex adaptive systems view of resilience in a project team. Systems Research and Behavioral Science 29 (5):499-516. http://dx.doi.org/10.1002/sres.2153

Fath, B. D. 2015. Quantifying economic and ecological sustainability. Ocean and Coastal Management 108:13-19. http:// dx.doi.org/10.1016/j.ocecoaman.2014.06.020

Fath, B. D., S. E. Jørgensen, B. C. Patten, and M. Straškraba. 2004. Ecosystem growth and development. Biosystems 77 (1-3):213-228. http://dx.doi.org/10.1016/j.biosystems.2004.06.001

Folke, C. 2006. Resilience: The emergence of a perspective for social-ecological systems analyses. Global Environmental Change 16(3):253-267. http://dx.doi.org/10.1016/j.gloenvcha.2006.04.002 
Folke, C., S. R. Carpenter, B. Walker, M. Scheffer, T. Chapin, and J. Rockström. 2010. Resilience thinking: integrating resilience, adaptability and transformability. Ecology and Society 15(4): 20. [online] URL: http://www.ecologyandsociety.org/vol15/iss4/ $\underline{\operatorname{art} 20 /}$.

Folke, C., T. Hahn, P. Olsson, and J. Norberg. 2005. Adaptive governance of social-ecological systems. Annual Review of Environment and Resources 30:441-473. http://dx.doi.org/10.1146/ annurev.energy.30.050504.144511

Geneske, J. 2013. Rebound: building a more resilient world. Rockefeller Foundation, New York, New York, USA. [online] URL: http://www.rockefellerfoundation.org/blog/rebound-buildingmore-resilient-world.

Gunderson, L. H. 2000. Ecological resilience: in theory and application. Annual Review of Ecology and Systematics 31:425-439. http://dx.doi.org/10.1146/annurev.ecolsys.31.1.425

Gunderson, L. H., and C. S. Holling, editors. 2002. Panarchy: understanding transformations in human and natural systems. Island Press, Washington, D.C., USA.

Hector, A., Y. Hautier, P. Saner, L. Wacker, R. Bagchi, J. Joshi, M. Scherer-Lorenzen, E. M. Spehn, E. Bazeley-White, M. Weilenmann, M. C. Caldeira, P. G. Dimitrakopoulos, J. A. Finn, K. Huss-Danell, A. Jumpponen, C. P. H. Mulder, C. Palmborg, J. S. Pereira, A. S. D. Siamantziouras, A. C. Terry, A. Y. Troumbis, B. Schmid, and M. Loreau. 2010. General stabilizing effects of plant diversity on grassland productivity through population asynchrony and overyielding. Ecology 91(8):2213-2220. http://dx. doi.org/10.1890/09-1162.1

Henry, D., and J. E. Ramirez-Marquéz. 2012. Generic metrics and quantitative approaches for system resilience as a function of time. Reliability Engineering and System Safety 99:114-122. http://dx. doi.org/10.1016/j.ress.2011.09.002

Holdschlag, A., and B. M. W. Ratter. 2013. Multiscale system dynamics of humans and nature in The Bahamas: perturbation, knowledge, panarchy and resilience. Sustainability Science 8 (3):407-421. http://dx.doi.org/10.1007/s11625-013-0216-6

Holling, C. S. 1973. Resilience and stability of ecological systems. Annual Review of Ecology and Systematics 4:1-23. http://dx.doi. org/10.1146/10.1146/annurev.es.04.110173.000245

Holling, C. S. 1986. The resilience of terrestrial ecosystems: local surprise and global change. Pages 292-317 in W. C. Clarkand and R. E. Munn, editors. Sustainable development of the biosphere: interactions between the world economy and the global environment. Cambridge University Press, Cambridge, UK.

Isbell, F. I., H. W. Polley, and B. J. Wilsey. 2009. Biodiversity, productivity and the temporal stability of productivity: patterns and processes. Ecology Letters 12:443-451. http://dx.doi. org/10.1111/j.1461-0248.2009.01299.x

Levin, S., T. Xepapadeas, A.-S. Crépin, J. Norberg, A. de Zeeuw, C. Folke, T. Hughes, K. Arrow, S. Barrett, G. Daily, P. Ehrlich, N. Kautsky, K.-G. Mäler, S. Polasky, M. Troell, J. R. Vincent, and B. Walker. 2013. Social-ecological systems as complex adaptive systems: modeling and policy implications. Environment and
Development Economics 18(2):111-132. http://dx.doi.org/10.1017/ $\underline{\mathrm{S} 1355770 \mathrm{X} 12000460}$

May, R. M., S. A. Levin, and G. Sugihara. 2008. Complex systems: ecology for bankers. Nature 451:893-895. http://dx.doi. org/10.1038/451893a

Nielsen, S. N., and R. E. Ulanowicz. 2011. Ontic openness: an absolute necessity for all developmental processes. Ecological Modelling 222(16):2908-2912. http://dx.doi.org/10.1016/j. ecolmodel.2011.05.012

O'Brien, G., and P. O'Keefe. 2010. Resilient responses to climate change and variability: a challenge for public policy. International Journal of Public Policy 6(3-4):369-385. http://dx.doi. org/10.1504/IJPP.2010.035136

Odum, H. T., and E. C. Odum. 2001. A prosperous way down: principles and policies. University Press of Colorado, Boulder, Colorado, USA.

Pelling, M., C. High, J. Dearing, and D. Smith. 2008. Shadow spaces for social learning: a relational understanding of adaptive capacity to climate change within organizations. Environment and Planning A 40(4):867-884. http://dx.doi.org/10.1068/a39148

Phelan, L., A. Henderson-Sellers, and R. Taplin. 2013. The political economy of addressing the climate crisis in the Earth system: undermining perverse resilience. New Political Economy 18(2):198-226. http://dx.doi.org/10.1080/13563467.2012.678820

Radywyl, N., and C. Biggs. 2013. Reclaiming the commons for urban transformation. Journal of Cleaner Production 50:159-170. http://dx.doi.org/10.1016/j.jclepro.2012.12.020

Ratter, B. M. W. 2013. Surprise and uncertainty-framing Regional Geohazards in the theory of complexity. Humanities 2 (1):1-19. http://dx.doi.org/10.3390/h2010001

Redman, C. L. 2014. Should sustainability and resilience be combined or remain distinct pursuits? Ecology and Society 19(2): 37. http://dx.doi.org/10.5751/ES-06390-190237

Robinson, L. W., and F. Berkes. 2011. Multi-level participation for building adaptive capacity: formal agency-community interactions in northern Kenya. Global Environmental Change 21:1185-1194. http://dx.doi.org/10.1016/j.gloenvcha.2011.07.012

Scheffer, M., F. Westley, W. A. Brock, and M. Holmgren. 2002. Dynamic interaction of societies and ecosystems: linking theories from ecology, economy and sociology. Pages 195-240 in L. H. Gunderson and C. S. Holling, editors. Panarchy: understanding transformations in human and natural systems. Island Press, Washington, D.C., USA.

Schweinberger, M., M. Petrescu-Prahova, and D. Q. Vu. 2014. Disaster response on September 11, 2001 through the lens of statistical network analysis. Social Networks 37:42-55. http://dx. doi.org/10.1016/j.socnet.2013.12.001

Standish, R. J., R. J. Hobbs, M. M. Mayfield, B. T. Bestelmeyer, K. N. Suding, L. L. Battaglia, V. Eviner, C. V. Hawkes, V. M. Temperton, V. A. Cramer, J. A. Harris, J. L. Funk, and P. A. Thomas. 2014. Resilience in ecology: abstraction, distraction, or where the action is? Biological Conservation 177:43-51. http://dx. doi.org/10.1016/j.biocon.2014.06.008 
Thomas, S., C. Keegan, S. Barry, R. Layte, M. Jowett, and C. Normand. 2013. A framework for assessing health system resilience in an economic crisis: Ireland as a test case. BMC Health Services Research 13: 450. http://dx.doi.org/10.1186/1472-6963-13-450

Ulanowicz, R. E., S. J. Goerner, B. Lietaer, and R. Gomez. 2009. Quantifying sustainability: resilience, efficiency and the return of information theory. Ecological Complexity 6(1):27-36.

Walker, B., S. Carpenter, J. Anderies, N. Abel, G. S. Cumming, M. Janssen, L. Lebel, J. Norberg, G. D. Peterson, and R. Pritchard. 2002. Resilience management in social-ecological systems: a working hypothesis for a participatory approach. Conservation Ecology 6(1): 14. [online] URL: http://www. consecol.org/vol6/iss1/art14/.

Walker, B., C. S. Holling, S. R. Carpenter, and A. Kinzig. 2004. Resilience, adaptability and transformability in social-ecological systems. Ecology and Society 9(2): 5. [online] URL: http://www. ecologyandsociety.org/vol9/iss2/art5.

Wilson, S., L. J. Pearson, Y. Kashima, D. Lusher, C. Pearson. 2013. Separating adaptive maintenance (resilience) and transformative capacity of social-ecological systems. Ecology and Society 18(1): 22. http://dx.doi.org/10.5751/ES-05100-180122 